\title{
A SIMPLE PROOF OF THE SPECTRAL CONTINUITY OF THE STURM-LIOUVILLE PROBLEM
}

\author{
PRZEMYSEAW KOSOWSKI \\ Institute of Mathematics, Polish Academy of Sciences \\ P.O. Box 13\%, 00-950 Warszawa, Poland \\ E-mail: kswsk@impan.gov.pl
}

\begin{abstract}
The aim of this article is to present a simple proof of the theorem about perturbation of the Sturm-Liouville operator in Liouville normal form.

1. Introduction. The purpose of this paper is to give a simple proof of the perturbation theorem concerning the spectral continuity of the Sturm-Liouville operator in normal form. The theorem in question can be stated as follows (see [6]): Let two problems in Liouville normal form $-u^{\prime \prime}+q_{i}(x) u=\lambda u \quad(i=1,2)$ be given on the same interval $[a, b]$ with the same boundary conditions. Let $\lambda_{k}^{(i)}(i=1,2)$ denote the $k$-th eigenvalues of the two problems $(k=1,2, \ldots)$. If $-\varepsilon \leq q_{2}(x)-q_{1}(x) \leq \varepsilon(\varepsilon>0)$ on $[a, b]$ then $-\varepsilon \leq \lambda_{k}^{(2)}-\lambda_{k}^{(1)} \leq \varepsilon$ for each $k=1,2, \ldots$ Instead of employing the Sturm comparison theorems for differential equations it is enough to use the Poincaré minmax principle. Another argument in the Hilbert space setting can be found in [4], Theorem V.4.10. The proof is based on the resolvent estimation and so it is also different from our approach.
\end{abstract}

2. Main result. Suppose that

$$
\begin{gathered}
D=\left\{u \in L^{2}([a, b]): u, u^{\prime} \text { absolutely continuous, } u^{\prime \prime} \in L^{2}([a, b])\right. \\
\text { and } \left.\alpha u(a)+\alpha^{\prime} u^{\prime}(a)=0, \beta u(b)+\beta^{\prime} u^{\prime}(b)=0\right\} .
\end{gathered}
$$

The constants $\alpha, \alpha^{\prime}, \beta, \beta^{\prime}$ are assumed to be real with $\alpha^{2}+\alpha^{\prime 2} \neq 0, \beta^{2}+\beta^{\prime 2} \neq 0$, and the interval $[a, b]$ is finite. For $u \in D$, let

$$
\begin{aligned}
& L u=-u^{\prime \prime}+q u, \\
& \tilde{L} u=-u^{\prime \prime}+\tilde{q} u,
\end{aligned}
$$

1991 Mathematics Subject Classification: 34B24, 34L15, 65L15, 65L60.

The paper is in final form and no version of it will be published elsewhere. 
where the functions $q, \tilde{q}$ are assumed to belong to $C([a, b])$. Now consider the eigenvalue problems for operators $L$ and $\tilde{L}$ :

$$
\begin{aligned}
& L u=\lambda u, u \in D, \\
& \tilde{L} u=\tilde{\lambda} u, u \in D .
\end{aligned}
$$

It is known that the operators $L$ and $\tilde{L}$ are self-adjoint (see [7]), and the eigensystems (1) and (2) with given separated boundary conditions are regular and have sequences of simple real distinct eigenvalues $\left\{\lambda_{k}\right\}_{k=1}^{\infty},\left\{\tilde{\lambda}_{k}\right\}_{k=1}^{\infty}$ such that

$$
\lambda_{1}<\lambda_{2}<\lambda_{3}<\ldots \quad \text { and } \quad \tilde{\lambda}_{1}<\tilde{\lambda}_{2}<\tilde{\lambda}_{3}<\ldots,
$$

and the corresponding sequences of orthonormal eigenfunctions $\left\{u_{k}\right\}_{k=1}^{\infty},\left\{\tilde{u}_{k}\right\}_{k=1}^{\infty}$ such that $\left\|u_{k}\right\|_{2}=\left\|\tilde{u}_{k}\right\|_{2}=1$ (see [1]). Now we are prepared to state the theorem.

Theorem. If $\|q-\tilde{q}\|_{\infty} \equiv \sup _{x \in[a, b]}|q(x)-\tilde{q}(x)| \leq \varepsilon$, then $\left|\lambda_{k}-\tilde{\lambda}_{k}\right| \leq \varepsilon$, for each $k=1,2,3, \ldots$

Proof. We have $-\varepsilon \leq q(x)-\tilde{q}(x) \leq \varepsilon$ for each $x$ in $[a, b]$. It is enough to consider only one inequality, e.g. the right one $q(x) \leq \tilde{q}(x)+\varepsilon, x \in[a, b]$. It is well known that each eigenvalue of the Sturm-Liouville problem satisfies Poincaré's minmax principle (see [2]), which asserts that

$$
\lambda_{k}=\min _{H_{k} \subset D} \max _{0 \neq u \in H_{k}} R[u],
$$

where $H_{k}$ denotes any $k$-dimensional subspace of $D$, and $R[u]$ is the Rayleigh quotient of (1), that is,

$$
R[u]=\frac{N[u]}{D[u]}
$$

where $N[u]=(L u, u)$ and $D[u]=(u, u)$. Integrating by parts we calculate

$$
N[u]=\int_{a}^{b}\left(-u^{\prime \prime}+q u\right) u d x=\int_{a}^{b}\left(\left(u^{\prime}\right)^{2}+q u^{2}\right) d x-\left[u u^{\prime}\right]_{a}^{b} .
$$

The boundary conditions are normal separated ones, so the problem is well posed. Precisely if we denote by $B C \equiv-\left[u u^{\prime}\right]_{a}^{b}$, then $B C$ can be expressed as $B C=B u^{2}(b)-A u^{2}(a)$, where the constants $A, B$ are real, whence

$$
\begin{aligned}
\lambda_{k} & =\min _{H_{k} \subset D} \max _{0 \neq u \in H_{k}} \frac{\int_{a}^{b}\left(\left(u^{\prime}\right)^{2}+q u^{2}\right) d x+B C}{\int_{a}^{b} u^{2} d x} \\
& \leq \min _{H_{k} \subset D} \max _{0 \neq u \in H_{k}} \frac{\int_{a}^{b}\left(u^{\prime}\right)^{2} d x+\int_{a}^{b}(\tilde{q}+\varepsilon) u^{2} d x+B C}{\int_{a}^{b} u^{2} d x}=\tilde{\lambda}_{k}+\varepsilon .
\end{aligned}
$$

Similarly we obtain that $\tilde{\lambda}_{k} \leq \lambda_{k}+\varepsilon$, whence $\left|\lambda_{k}-\tilde{\lambda}_{k}\right| \leq \varepsilon$. This completes the proof.

COROLlary. Suppose that the function $q$ in (1) depends on a parameter $t$ with Lipschitz constant $C>0$, so $|q(x, s)-q(x, t)| \leq C|s-t|, x \in[a, b], s, t \in \mathbb{R}$. Then each $\lambda_{k}$ depends continuously on $t$ with the same Lipschitz constant. 
Proof. For fixed $s$ and $t$ it is enough to take $\varepsilon=C|s-t|$ and apply the Theorem, obtaining thus

$$
\left|\lambda_{k}(s)-\lambda_{k}(t)\right| \leq C|s-t| \quad \forall k=1,2, \ldots
$$

Now we give three examples.

EXAmPle 1. Consider the perturbation of the equation $-u^{\prime \prime}=\lambda u, u(0)=u(\pi)=0$ to $-u^{\prime \prime}+\varepsilon \frac{x}{\pi} u=\lambda u$ with the same boundary conditions $u(0)=u(\pi)=0$. For the unperturbed Sturm-Liouville problem we have the eigenvalues $\lambda_{k}=k^{2}$ and the eigenfunctions $u_{k}(x)=\sqrt{\frac{2}{\pi}} \sin k x \quad(k=1,2, \ldots)$. Let $q_{\varepsilon}(x)=\varepsilon \frac{x}{\pi}$ and $q_{0}(x)=0$. We compute the approximation of the smallest eigenvalue $\lambda_{1}^{\varepsilon}$ of the perturbed equation. To do this we use the finite element method with the basis of hat functions with 1200 nodes in $[0, \pi]$. This method gives us the accuracy up to 5 digits after the period. The calculations were made on PC486 computer with floating point arithmetic for the real type. Results are presented in the table given below:

\begin{tabular}{||c||c||c||c||}
\hline$\varepsilon$ & $\lambda_{1}^{\varepsilon}$ & $\left\|q_{\varepsilon}-q_{0}\right\|_{\infty}$ & $\lambda_{1}^{\varepsilon}-\lambda_{1}$ \\
\hline \hline 1 & 1.48919 & 1 & 0.48919 \\
\hline 0.1 & 1.04989 & 0.1 & 0.04989 \\
\hline 0.2 & 1.09956 & 0.2 & 0.09956 \\
\hline 0.01 & 1.00499 & 0.01 & 0.00499 \\
\hline
\end{tabular}

EXAMPLE 2. In this example we consider two equations $-u^{\prime \prime}+q_{i}(x) u=\lambda u \quad(i=1,2)$ on the interval $[0, \pi]$, where $q_{1}(x)=0.1 \cdot \frac{x}{\pi}$ and $q_{2}(x)=0.1 \cdot\left(\frac{x}{\pi}\right)^{2}$ with the same boundary conditions $u(0)=u(\pi)=0$. We compute the first three eigenvalues of these equations using the method described in Example 1. Results are presented in the table given below:

\begin{tabular}{||c||c||c||c||c||}
\hline$k$ & $\lambda_{k}^{(1)}$ & $\lambda_{k}^{(2)}$ & $\lambda_{k}^{(1)}-\lambda_{k}^{(2)}$ & $\left\|q_{1}-q_{2}\right\|_{\infty}$ \\
\hline \hline 1 & 1.04989 & 1.02815 & 0.02174 & 0.025 \\
\hline 2 & 4.05003 & 4.03210 & 0.01794 & 0.025 \\
\hline 3 & 9.05001 & 9.03283 & 0.01723 & 0.025 \\
\hline
\end{tabular}

EXAMPLE 3 . If we consider the perturbed equation $-u^{\prime \prime}+\varepsilon u=\lambda u$ with boundary conditions $u(0)=u(\pi)=0, \varepsilon>0$ we obtain eigenvalues $\lambda_{k}^{\varepsilon}=k^{2}+\varepsilon$. Further $\left\|q_{\varepsilon}-q_{0}\right\|_{\infty}=$ $\varepsilon$, and so $\left|\lambda_{k}^{\varepsilon}-\lambda_{k}\right|=\varepsilon=\left\|q_{\varepsilon}-q_{0}\right\|_{\infty}$.

Conclusion. These three examples show that the inequality in the Theorem can be strict (like in Examples 1, 2; for eigenvalue and eigenfunction asymptotics for a regular Sturm-Liouville operator see [3], [5]) or it just becomes equality (like in Example 3). In this sense the result of the Theorem is sharp.

3. Extensions and generalizations. The idea of our proof can be extended to higher dimensions, that is the following spectral continuity problem in two dimensions can be considered: $-\Delta u+q u=\lambda u$ in $\Omega \subset \mathbb{R}^{2}$ and $u=0$ on $\partial \Omega$.

For numerical analysis, the inequality in the Theorem gives an estimation of the absolute error of eigenvalues $\lambda_{k}$ for the eigenvalue problem (1) when the data (function $q$ ) 
are perturbed. There is a natural question about the relative error of $\lambda_{k}$, that is, an estimation of $\left|\frac{\tilde{\lambda}_{k}-\lambda_{k}}{\lambda_{k}}\right|$. The author intends to consider this question in another paper.

Acknowledgements. The author is grateful to Dr. Alicja Smoktunowicz and Professor Jaroslav Zemánek for helpful discussions and valuable guidance.

\section{References}

[1] G. Birkhoff and G.-C. Rota, Ordinary Differential Equations, Ginn-Blaisdell, Boston, 1962.

[2] R. Courant and D. Hilbert, Methods of Mathematical Physics, vol. 1, Interscience, New York, 1953.

[3] C. T. Fulton and S. Pruess, Eigenvalue and eigenfunction asymptotics for regular SturmLiouville problems, J. Math. Anal. Appl. 188 (1994), 297-340.

[4] T. Kato, Perturbation Theory for Linear Operators, Springer-Verlag, Berlin, 1966.

[5] B. M. Levitan and I. S. Sargsyan, Sturm-Liouville and Dirac Operators, Kluwer, Dordrecht, 1991.

[6] J. D. Pryce, Numerical Solution of Sturm-Liouville Problems, Clarendon Press, New York, 1993.

[7] M. H. Stone, Linear Transformations in Hilbert Space, American Mathematical Society, New York, 1932. 